\title{
Abnormal expression of HOXD11 promotes the malignant behavior of glioma cells and leads to poor prognosis of glioma patients
}

\author{
Jialin Wang Equal first author, 1, ${ }^{2}$, Zhendong Liu Equal first author, ${ }^{3}$, Cheng Zhang ${ }^{4}$, Hongbo Wang ${ }^{5}$, Ang Li $^{3}$, Binfeng Liu $^{1}$, Xiaoyu \\ Lian $^{1}$, Zhishuai Ren ${ }^{1}$, Wang Zhang ${ }^{6}$, Yanbiao Wang ${ }^{1}$, Bo Zhang ${ }^{5}$, Bo Pang ${ }^{7}$, Yanzheng Gao ${ }^{\text {Corresp. } 3}$

Background. Homeobox D11 (HOXD11) plays an important role in a variety of cancers, but its precise role in gliomas remains unclear. This study aimed to explore the relationship between HOXD11 and gliomas by combining bioinformatics methods with basic experimental validation.

Materials and methods. Obtain gene expression information and clinical information of glioma and non-tumor brain tissue samples from multiple public databases such as TCGA (666 glioma samples), CGGA (749 glioma samples), GEPIA(163 glioblastoma samples and 207 normal control samples), GEO (GSE4290 and GSE15824). 9 cases of glioma tissue and 5 cases of normal control brain tissue were collected from the clinical department of Henan Provincial People's Hospital for further verification. A series of bioinformatic analysis methods were used to confirm the relationship between HOXD11 expression and overall survival and clinical molecular characteristics of patients with glioma. RT-qPCR was used to verify the change of expression level of HOXD11 in glioma cells and tissues. MTT assay, colony formation assay, wound-healing assay, immunofluorescence staining, flow cytometry and western blotting were used to detect the effect of HOXD11 on the biological behavior of glioma cell line U251.

Results. The high expression of HOXD11 was significantly related to age, World Health Organization (WHO) grade, chemotherapy status, histological type, and even 1p19q codeletion data and isocitrate dehydrogenase (IDH) mutation. HOXD11, as an independent risk factor, reduces the overall survival of glioma patients and has diagnostic value for the prognosis of glioma. Gene Set Enrichment Analysis (GSEA) showed that HOXD11 was significantly enriched in cell signaling pathway such as cell cycle, DNA replication and so on. Finally, we confirmed that the knockout of HOXD11 can inhibit the proliferation and invasion of U251 glioma cells, and change the biological behavior of tumor cells by preventing the progression of cell cycle.

Conclusions. HOXD11 may be used as a candidate biomarker for the clinical application of targeted drug and prognostic assessment treatment of glioma. In addition, This study will help to explore the

Peer] reviewing PDF | (2020:09:53246:1:1:CHECK 15 Dec 2020) 
pathological mechanism of glioma. 


\section{Abnormal expression of $H O X D 11$ promotes the malignant 2 behavior of glioma cells and leads to poor prognosis of 3 glioma patients}

4 Jialin Wang ${ }^{1,2 *}$, Zhendong Liu ${ }^{3 *}$, Cheng Zhang ${ }^{4}$, Hongbo Wang ${ }^{5}$, Ang $\mathrm{Li}^{3}$, Binfeng Liu ${ }^{1}$, Xiaoyu

5 Lian ${ }^{1}$, Zhishuai Ren ${ }^{1}$, Wang Zhang ${ }^{6}$, Yanbiao Wang ${ }^{1}$, Bo Zhang ${ }^{5}$, Bo Pang 7 , YanZheng Gao ${ }^{3 \&}$

$6{ }^{1}$ Zhengzhou University People's Hospital, Henan Provincial People's Hospital, Zhengzhou,

7 Henan, China

$8 \quad{ }^{2}$ Department of Microbiome Laboratory, Henan Provincial People's Hospital, People's Hospital

9 of Zhengzhou University, Zhengzhou University, Zhengzhou, Henan, China

$10{ }^{3}$ Department of Surgery of Spine and Spinal Cord, Henan Provincial People's Hospital, People's

11 Hospital of Zhengzhou University, School of Clinical Medicine, Henan University, Zhengzhou,

12 Henan, China

$13 \quad{ }^{4}$ North broward preparatory school, Nord Anglia Education, Coconut Creek, Florida, United

14 States

$15{ }^{5}$ Henan University People's Hospital, Henan Provincial People's Hospital, Zhengzhou, Henan,

16 China

$17{ }^{6}$ Department of neurosurgery of the First affiliate Hospital of Harbin Medical University,

18 Harbin, Heilongjiang, China.

19 7. Department of Neurosurgery, The Fourth Medical Center of Chinese PLA General Hospital, 
Beijing, China

21 \& Correspondence: 13937139998@163.com

* These authors contributed equally to this work.

\section{ABSTRACT}

Background. Homeobox D11 (HOXD11) plays an important role in a variety of cancers, but its precise role in gliomas remains unclear. This study aimed to explore the relationship between HOXD11 and gliomas by combining bioinformatics methods with basic experimental validation.

Materials and methods. Obtain gene expression information and clinical information of glioma and non-tumor brain tissue samples from multiple public databases such as TCGA (666 glioma samples), CGGA (749 glioma samples), GEPIA(163 glioblastoma samples and 207 normal control samples), GEO (GSE4290 and GSE15824). 9 cases of glioma tissue and 5 cases of normal control brain tissue were collected from the clinical department of Henan Provincial People's Hospital for further verification. A series of bioinformatic analysis methods were used to confirm the relationship between $H O X D 11$ expression and overall survival and clinical molecular characteristics of patients with glioma. RT-qPCR was used to verify the change of expression level of HOXD11 in glioma cells and tissues. MTT assay, colony formation assay, wound-healing assay, immunofluorescence staining, flow cytometry and western blotting were used to detect the effect of $H O X D 11$ on the biological behavior of glioma cell line U251.

Results. The high expression of $H O X D 11$ was significantly related to age, World Health 
41 Organization (WHO) grade, chemotherapy status, histological type, and even 1p19q codeletion

data and isocitrate dehydrogenase (IDH) mutation. HOXD11, as an independent risk factor, reduces the overall survival of glioma patients and has diagnostic value for the prognosis of glioma. Gene Set Enrichment Analysis (GSEA) showed that HOXD11 was significantly enriched in cell signaling pathway such as cell cycle, DNA replication and so on. Finally, we confirmed that the knockout of $H O X D 11$ can inhibit the proliferation and invasion of U251 glioma cells, and change the biological behavior of tumor cells by preventing the progression of cell cycle. Conclusions. HOXD11 may be used as a candidate biomarker for the clinical application of targeted drug and prognostic assessment treatment of glioma. In addition, This study will help to explore the pathological mechanism of glioma.

INTRODUCTION

Gliomas are one of the most common primary intracranial neoplasms, accounting for $81 \%$ of intracranial neoplasms(Ostrom et al. 2014). The treatment options for glioma are mainly focused on maximizing surgical removal of the tumor tissue and assisting in comprehensive radiotherapy and chemotherapy (Taal et al. 2015). The postoperative recurrence and poor prognosis of gliomas remain a major problem. Therefore, scientists have adopted new treatment methods, such as immunotherapy and photodynamic therapy, to overcome current limitations (Kong et al. 2018; Zavadskaya capital Te 2015). However, these new treatments have not yet completely improved the prognosis of gliomas. Part of the reason for this may be that the pathogenesis of glioma has not been fully elucidated. 
In recent years, many studies have reported that epigenetics plays a crucial role in the

development and regulation of cancer (Dawson \& Kouzarides 2012). Epigenetics is involved in regulating the pathophysiological process of tumors. Epigenetics is a general term for various modifications that do not change the sequence of genetic material but can lead to changes in gene expression, such as methylation, acetylation and phosphorylation (Abbaoui et al. 2017; Jablonska \& Reszka 2017; Kondo et al. 2017; Zhang et al. 2017). Significant advances have been made in the field of epigenetics in recent years, mainly due to the rapid development of high-throughput whole-genomes sequencing that provide researchers with all genetic expression changes associated with genetic modification (Kim et al. 2016).

TCGA is a widely used public database storing the genetic and clinical information of a variety of human malignant tumors, and it has made great contributions to the research on human malignant tumors(Tomczak et al. 2015). In addition, CGGA is a free database dedicated to glioma research. It also stores a large sample of gene expression profile information and patientrelated clinical information, which may help researchers explore the relationship between genetic information of gene expression and clinical data related to glioma. Therefore, researchers' understanding of CGGA-based glioma is gradually expanding(Liang et al. 2019; Wang et al. 2018). For example, Feng et al. found that high expression of major histocompatibility complex, class I, F (HLA-F) can predict poor prognosis in patients with glioma (Feng et al. 2019). Guan et al. showed the abnormally high expression of CKLF like MARVEL Transmembrane domain containing 6 (CMTM6) is involved in the pathophysiological process to promote poor prognosis of glioma (Guan et al. 2018). Therefore, our study attempts to find a biomarker related to the 
83

84

85

prognosis of glioma through the combination of bioinformatics method and traditional experimental verification.

HOXD11 belongs to the HOX gene family, which encodes transcription factors that regulate various physiological processes. In recent years, many articles have reported that $H O X D 11$ plays a vital regulatory role in tumors like laryngeal squamous cell carcinoma, ovarian cancers, head and neck cancer, and others (Cai et al. 2007; de Barros et al. 2016; Sharpe et al. 2014). However, as far as we know, there is no report on the relationship between HOXD11 and glioma. Here, we examined the relationship between high or low expression level of HOXD11 and the prognosis and clinical features of glioma using bioinformatics approaches. Further indirect analysis by Gene Set Enrichment Analysis (GSEA) was used to reveal the mechanism of action of HOXD11 in gliomas. Finally, a variety of experimental methods have been used to confirm that HOXD11 can promote the malignant behavior of glioma cells as an oncogene by participating in the regulation of cell cycle signaling pathways. Thus, we believe this study will provide a new diagnostic biomarker for the diagnosis and therapeutic target for gliomas.

MATERIAL AND METHODS

\section{Data collection}

GEPIA is an integrated database platform, which is characterized by the fusion of gene sequencing data of tumor samples from TCGA database and gene sequencing data of normal control group sample tissues from The Genotype-Tissue Expression (GTEx) (Tang et al. 2017). This advantage of GEPIA makes up for the shortage of normal control samples in TCGA 
104

105

106

107

108

109

110

111

112

113

114

115

116

117

118

119

120

121

122

123

124

database. The expression levels of $H O X D 11$ in various tumors and tissues, including 163

glioblastoma, 518 low grade glioma and 207 normal brain tissue samples, were obtained from the GEPIA database. To further determine the expression level changes of HOXD11 in glioma, we obtained gene expression data in the form of FPKM (Fragments Per Kilobase per Million) from a glioma tissue microarray (GSE4290) and a glioma cell microarray (GSE15824) from GEO database (https://www.ncbi.nlm.nih.gov/geo/) for further analysis. GSE4290 contains 23 non-tumor brain tissue samples and 77 glioma tissue samples(Sun et al. 2006). GSE15824 contains three human-derived astrocyte (HA) cell line samples and two LN319, two LN229, two BS149 and two LN018 glioblastoma cell line samples(Grzmil et al. 2011).

CGGA (http://www.cgga.org.cn/) is a public database focused on glioma research, which contains many types of data and corresponding clinical information(Guan et al. 2018). This part of the data contains a variety of clinical information of the patients and was used to further depth analyze the relationship between changes in $H O X D 11$ expression level and glioma prognosis. We examined two RNA-seq datasets containing 693 and 325 samples. After excluding the data of patients with incomplete clinical data, we obtained 749 glioma samples for further analysis of various types.

In order to verify the effect of $H O X D 11$ on the prognosis and diagnostic value of glioma, we searched and obtained one RNA-seq dataset containing 666 glioma tissues in the TCGA database (https://portal.gdc.cancer.gov/) and obtained clinical information of the corresponding patients. Nine glioma tissue samples and five non-tumor brain tissue samples were collected and stored in a liquid nitrogen environment during surgery and then transferred to $\mathrm{a}-80^{\circ} \mathrm{C}$ freezer 
125

126

127

128

129

130

131

132

133

134

until use. Clinical samples were obtained after receiving written informed consent from patients in the clinical department of Henan Provincial People's Hospital. This study has been approved by the Institutional Review Board of Zhengzhou University.

\section{GSEA analysis of $\mathrm{HOXD11}$}

GSEA is widely used as a bioinformatics analysis tool for gene function annotation and analysis. The RNA-seq data obtained from the CGGA database was batch-corrected and normalized by limma packages and then classified into "group H" (high expression group) or "group L"(low expression group) based on the median expression level of $H O X D 11$. Functional enrichment analysis was performed using the GSEA 4.0.2 jar software. The number of permutations was set to 1000 times, and the gene set database was set to the Kyoto Encyclopedia of Genes and Genomes (KEGG) cell signaling pathway.

\section{Cell culture}

Human glioma cell lines (U251, LN229 and T98) and human-derived astrocyte (HA) were purchased from the Cell Bank of the Chinese Academy of Sciences (Shanghai, China). All cells were grown in incubators at $37^{\circ} \mathrm{C}$ and $5 \%$ carbon dioxide and cultured using DMEM medium (Thermo Fisher Scientific, USA) plus 10\% FBS (Thermo Fisher Scientific, USA).

RNA extraction and Quantitative Reverse Transcription Polymerase Chain Reaction (RT-qPCR) analysis 
1469 glioma tissue and 5 peritumoral tissue brain tissue samples were used to detect the expression

147 level of HOXD11 in tissues by RT-qPCR. Three glioma cell lines (T98, U251, LN229) and one

148 human astrocyte were used to detect the expression level of HOXD11 in glioma cells. First, total

149 RNA was extracted from glioma and normal brain tissue samples using Tri-Reagent (Sigma,

150 USA). The quality and quantity assessments of total RNA were determined using NanoDrop One

151 spectrophotometer (Thermo Fisher Scientific, USA), evaluating 260/280 nm absorbance values.

152 Thereafter, the cDNA was reverse transcribed from the total RNA using the Transcriptor First

153 Strand cDNA Synthesis kit (Roche, USA). RT-qPCR was performed following the guidelines for

154 FastStart Universal SYBR Green Master (ROX) (Roche, Germany). Results were quantified

155 using QuantStudio software (Thermo Fisher Scientific, USA) following the manufacturer's

instructions. GADPH was used as an internal reference. The primer sequences used for HOXD11

were 5'-GAACGACTTTGACGAGTGCG-3' (F) and 5'-ACGGTTGGGAAAGGAACGAA-3'

158

(R). The expression level of $H O X D 11$ was measured by the "2- $-\Delta \triangle \mathrm{CT}$ " method. Statistical

differences between the two groups were analyzed by unpaired t-test, and results were

considered statistically significant when the $\mathrm{p}$ value was $<0.05$.

\section{Cell transfection}

The small interfere RNA (siRNA) that specifically targeting HOXD11 was purchased from

Genepharma (Shanghai, China). The siRNA sequence was sense(5'- 
167 and antisense, (5'-ACGUGACACGUUCGGAGAATT-3'). Cells were transfected using

168 Lipofectamine 2000 reagent (Invitrogen, Carlsbad, CA, USA). Detection of transfection

169 efficiency by RT-qPCR at 48 hours after transfection.

170

171

\section{Cell proliferation assay}

To detect the effect of HOXD11 on the proliferation ability of U251 cells, MTT experiments were conducted. The transfected cells were transferred into $96-w e l l$ plates $\left(1 \times 10^{3}\right.$ cells/well $)$. Cell proliferation ability was measured at 12 hours, 24 hours, 48 hours and 96 hours after transfection. The specific method was to add $20 \mathrm{ul}$ of MTT $(5 \mathrm{mg} / \mathrm{ml})$ to each well for culture. The liquid was discarded after incubation for 4 hours and 150 ul DMSO was added to each well. After 15 minutes of shaking, the optical density (OD) of $490 \mathrm{~nm}$ was detected using microplate reader.

\section{Colony formation assay}

In colony formation assay, U251 cells were planted in six-well plates at a density of about 300 cells/well. After 10 days of culture, the cells were fixed with 4\% paraformaldehyde and stained with $0.1 \%$ crystal violet. Colonies larger than $1 \mathrm{~mm}$ in diameter were counted using ImageJ Software(v1.8.0) after photographing.

\section{Wound-healing assay}

The exponential growth cells were seeded on the 6-well plate with a fusion degree of $90 \%$. A 
straight linear scratch was made on the center of the plate with a pipette (200uL). PBS was washed gently, and serum-free medium was replaced for culture. After that, the scratch width was observed and photographed under the microscope for 24 hours and 48 hours respectively, then was measured by ImageJ Software (v1.8.0).

\section{Immunofluorescence staining}

Firstly, treated cells were fixed with $4 \%$ paraformaldehyde for 30 minutes, and then $0.3 \%$ Triton X-100 was used to penetrate for 20 minutes at room temperature. After blocking with 5\% BSA, primary antibody Ki67 $\left(1: 1000\right.$, Abcam, UK) was added and placed in a $4^{\circ} \mathrm{C}$ refrigerator overnight. After that, washed 3 times with PBS for 5 minutes each time. Secondary antibody was added and incubated for one hour at room temperature in the dark. After washing with PBS, DAPI was added for nucleation. The photographs were finally observed using a fluorescence microscope. After that, the total number of cells and the number of KI67 positive cells were counted by ImageJ Software (v1.8.0).

\section{Flow cytometry}

U251 cells were digested and centrifuged to collect the cells after transfection. Precooled 75\% ethanol was added, fixed overnight at $-20^{\circ} \mathrm{C}$ Each tube was added with $500 \mathrm{uL}$ propidium iodide (PI) dye solution, stained for 30 minutes in dark, and filtered through a filter. Cell cycle distribution was analyzed by flow cytometry (Canto plus, BD, USA). 
209

210

211

212

213

214

215

216

217

218

219

220

221

222

223

224

225

226

227

228

229

\section{Western blotting}

Total protein of transfected U251 cells was extracted using RIPA buffer with protease inhibitor.

Separated by $10 \%$ SDS-PAGE gel electrophoresis, transferred to polypropylene fluoride

membrane (PVDF) treated with methanol for 2 hours, transferred to a shaker, sealed with TBS-T

blocking solution containing 5\% skimmed milk powder for 1 hours. Then, the primary antibodies

GAPDH (1:5000, Abcam, UK), CCNB1 (1:500, Abcam, UK), CDK1 (1:500, Abcam, UK) and

Cdc25C (1:500, Abcam, UK) were added to incubate overnight at $4^{\circ} \mathrm{C}$. Washed by TBS-T 5

times for 5 minutes each time, secondary antibody (1:5000) was added to incubate for 1 hours at room temperature, Washed by TBS-T 5 times for 5 minutes each time, and finally ECL color developing solution was added for color development. The developed bands were subjected to gray level analysis by ImageJ Software (v1.8.0), The difference in protein expression levels between the HOXD11-NC group and the HOXD11-siRNA group is represented by the gray value of the corresponding protein bands.

\section{Statistical analysis}

R software (v.3.6.1 version) and GraphPad Prism (v8.0.2) was used to process data and perform statistical analysis. Unpaired t-test was used for comparison between the two groups. The expression levels of $H O X D 11$ were examined in glioma and non-tumor brain tissue samples by the Wilcox test method. The relationship between the Overall Survival rate of patients and the expression level of $H O X D 11$ was analyzed by the Kaplan-Meier method and Cox regression 
230

231

232

233

234

235

236

237

238

239

240

241

242

243

244

245

246

247

248

249

250

analysis, and the survival curve was drawn accordingly. The relationship between clinically relevant patient information and the expression level of $H O X D 11$ was analyzed using the Wilcox and Kruskal tests.

\section{Results}

\section{Abnormally high expression of $\mathrm{HOXD11}$ in various tumors including gliomas}

Through GEPIA database, we found that $H O X D 11$ was abnormally highly expressed in a variety of tumors, such as ESCA, GBM, HNSC and HUSC, especially in GBM, as shown in Figure 1a. To confirm the expression level of HOXD11 in gliomas relative to normal controls, data from more sources were analyzed. By analyzing the data of glioma tissues and cell lines microarrays obtained from GEO database, it was found that $H O X D 11$ was highly expressed in both glioma tissues and cell lines, as shown in Figure $1 \mathrm{~b}$ and $1 \mathrm{c}$. To validate the above analysis results, we performed RT-qPCR at tissue and cell levels, respectively. The results showed that HOXD11 was highly expressed in glioma tissues and cell lines compared with normal brain tissues and human astrocytes, as shown in Figures 1d and 1e. Among them, the relevant clinical information of 9 glioma tissue samples was shown in Table S1.

\section{Correlation between the expression level of $H O X D 11$ and the clinical and prognosis of patients with} glioma

To clarify the relationship between high expression of HOXD11 and the clinical and prognosis of glioma patients, we conducted further studies using glioma sequencing data and relevant clinical 
251

252

256

257

258

259

260

261

262

263

264

265

266

267

268

269

270

271

information from CGGA database. A total of 749 glioma samples with complete clinical

information were obtained from the CGGA database, which contains a variety of clinical

information such as Primary-Recurrent-Secondary (PRS) type, age (age at diagnosis), gender,

histological type, and chemotherapy status, as well as $1 \mathrm{p} 19 \mathrm{q}$ codeletion status and isocitrate IDH

mutation status. More detailed information of clinical characteristics is shown in Table S2.

The Wilcox and Kruskal tests were used to analyze the relationship between clinically

relevant patient information and the expression level of $H O X D 11$. The expression level of

HOXD11 was significantly correlated with PRS type, WHO grade, chemotherapy status, age,

1p19q codeletion data, IDH mutation status and histological type (Figure $2 \mathrm{a}-\mathrm{g}$ ). The expression

level of HOXD11 increases as the WHO grade and age of the glioma increases $(\mathrm{p}<0.001)$. As

for the IDH mutation status, there was a higher level of gene expression in wildtype than in the

mutant $(\mathrm{p}<0.001)$. Moreover, for $1 \mathrm{p} 19 \mathrm{q}$ codeletion status, there were lower gene expression

levels in patients with a codeletion of $1 \mathrm{p} 19 \mathrm{q}$ than in patients with a non-codeletion of $1 \mathrm{p} 19 \mathrm{q}(\mathrm{p}<$ $0.001)$.

\section{Survival outcomes of $H O X D 11$ in patients with glioma}

The relationship between overall survival and the expression level of HOXD11 in patients with gliomas was explored by Kaplan-Meier survival analysis. The 749 patients with gliomas included in the study were divided into two groups: "group H" and "group L," based on the median expression level of $H O X D 11$. As shown in Figure 3a, the Overall Survival (OS) was significantly lower in patients with gliomas having high expression levels of HOXD11 (group H) 
272

273

274

275

276

277

278

279

280

281

282

compared to those with low levels (group L; $<<0.001$ ) in CGGA-sequence, which indicated poor prognosis.

To improve the credibility of the data results, we further obtained 666 glioma tissues in the TCGA database. The Kaplan-Meier method was used to verify that the increase of HOXD11 expression level can indeed reduce the overall survival time of glioma patients (Figure 3b). To clarify the impact of $H O X D 11$ expression level on the prognosis of gliomas of different molecular subtypes, we divided the samples into three groups (IDH mutation with 1p19q codeletion, IDH mutation without 1p19q codeletion and IDH wild type) for survival analysis.

The results showed that the prognosis of the group with high HOXD11 expression was significantly worse than that of the group with low HOXD11 expression among the three molecular subtypes, as shown in Figure 3 c-e. These results suggest that high expression of HOXD11 may lead to poor prognosis of patients with glioma.

\section{High expression of $H O X D 11$ is an independent risk element in patients with gliomas}

To determine whether HOXD11 could be used as an independent prognostic factor, we performed univariate and multivariate analyses for the date from CGGA-sequence. Univariate analysis was performed by applying Cox regression model (Figure 4a). The results suggested that high expression levels of $H O X D 11$ was significantly related to poor prognosis as a risk factor for glioma $(\mathrm{p}<0.001 ; \mathrm{HR}=1.550 ; 95 \% \mathrm{CI}, 1.456-1.650)$. The PRS type, histology, WHO grade, age and other factors showed similar results. Next, multivariate analysis was performed using the Cox regression model (Figure 4b). High expression of $H O X D 11$ ( $\mathrm{p}<0.001$; 
$\mathrm{HR}=1.156 ; 95 \% \mathrm{CI}, 1.072-1.246), \mathrm{PRS}$ type $(\mathrm{p}<0.001 ; \mathrm{HR}=1.947 ; 95 \% \mathrm{CI}, 1.655-2.291)$,

and high WHO grade $(\mathrm{p}<0.001 ; \mathrm{HR}=2.673 ; 95 \% \mathrm{CI}, 1.955-3.655)$ were significantly

associated with poor prognosis. Together, the univariate and multivariate analyses results suggest that high expression levels of $H O X D 11$ may be an independent risk factor for poor prognosis.

High expression of $\mathrm{HOXD} 11$ has certain evaluation value for glioma prognosis

To clarify the diagnostic value of highly expressed $H O X D 11$ for glioma prognosis, we plotted

the receiver operating characteristic (ROC) curve based on the sequencing data of glioma

patients from CGGA and TCGA databases. As shown in Figure 4c-d, the AUC values were

greater than 0.700 in each dataset, indicating that high expression of $H O X D 11$ had certain

evaluation value for one-year, three-year and five-year survival rates. These results indicate that

HOXD11 expression level could be related to prognosis in patients with gliomas; moreover, high expression of $H O X D 11$ may be used for clinical prognosis evaluation of patients with glioma.

Knockout of $H O X D 11$ could significantly inhibit the proliferation ability of $\mathrm{U} 251$ cells

By analyzing the sequencing data of glioma samples and matching clinical information from

multiple sources, the results showed that $H O X D 11$ could be an independent prognostic factor for glioma and had certain diagnostic value. To verify the effect of $H O X D 11$ on malignant

biological behavior of glioma cell lines, we conducted a series of phenotypic experiments. First,

312 we used siRNA to interfere with the expression level of HOXD11 in glioma cell U251.

Meanwhile, the transfection efficiency was detected by RT-qPCR (Figure 5a).To determine the 
314 effect of HOXD11 on the proliferation ability of U251 cells, we performed MTT assay. The

315 results showed that the proliferation ability of $H O X D 11$ knockdown group $(H O X D 11$-siRNA

316 group) was significantly reduced compared with the control group(HOXD11-NC) (Figure. 5e).

317 Meanwhile, HOXD11 knockdown also reduced the ability of cell colony formation (Figure. 5b).

318 Meanwhile, immunofluorescence staining results showed that the expression level of cell

319 proliferation marker KI67 in the knockdown group was significantly lower than that in the

320

321

322

323

324

325

326

327

328

329

330

331

332

333

334

control group (Figure 5c,d). In addition, similar results were obtained in the wound-healing assay, and the wound healing ability was also significantly reduced in the HOXD11 knockdown group (Figure. 5f).Therefore, we believe that highly expressed HOXD11 is involved in the pathological process of glioma by affecting the proliferation and migration of glioma cells.

\section{Determination of $\mathrm{HOXD11-related} \mathrm{cellular} \mathrm{signaling} \mathrm{pathway} \mathrm{by} \mathrm{GSEA}$}

To clarify how HOXD11 plays a role in the pathological process of glioma, we performed GSEA analysis to predict the cell signaling pathways that HOXD11 may participate in. As shown in Table 1 and Figure 6a, HOXD11 may be involved in a variety of cancer-related cell signaling pathways such as cell cycle, DNA replication, ECM receptor interaction, and focal adhesion. These results reveal that $H O X D 11$ may be involved in these cancer-related cell signaling pathway as an oncogene.

High expression of $\mathrm{HOXD11}$ participates in the pathological process of glioma by regulating cell cycle Through GSEA analysis, we predicted that highly expressed $H O X D 11$ may participate in the 
335

336

337

338

pathological process of glioma through a variety of cancer-related cell signaling pathways.

We further chose cell cycle signaling pathway as an object to verify the prediction results. To explore the effect of HOXD11 on the cell cycle of glioma cell U251, we performed flow cytometry. The results showed that in the HOXD11 knockdown group, U251 cells were significantly blocked in G2 phase compared with the control group (Figure. 6b). To validate this result, we performed western blotting. The results showed that the expression levels of cell cycle-related proteins (CCNB1, CDK2 and Cdc25c) were significantly reduced in $\mathrm{U} 251$ cells with $H O X D 11$ knockdown, confirming that $H O X D 11$ is involved in regulating cell cycle progression (Figure. 6c).

\section{Discussion}

The results of our study indicate that $H O X D 11$ is highly expressed in glioma samples relative to non-tumor brain tissue. In addition, high expression of $H O X D 11$ may be associated with poor prognosis in glioma patients. Moreover, ROC analysis verified the diagnostic value of HOXD11. These results indicate that $H O X D 11$ may be used for the treatment, prognosis evaluation and prediction of glioma as a biomarker.

Numerous studies have shown that HOXD11 is involved in tumor development and that it helps regulate gene expression. Xu et al. showed that abnormal methylation of HOXD11 may be related to lung cancer pathology (Xu et al. 2019). At the same time, studies have suggested that aberrant methylation of $H O X D 11$ can be used to assess risk stratification and early diagnosis of lung cancer as a biomarker (Harada et al. 2019). Studies have suggested that HOXD11 is highly 
356

357

358

359

360

361

362

363

364

365

366

367

368

369

370

371

372

373

374

375

376

expressed in Oral Squamous Cell Carcinoma (OSCC) as a homeobox gene (Rodini et al. 2012).

In addition, $H O X D 11$ may participate in the progression of laryngeal squamous cell carcinoma

by promoting cell proliferation and cell migration (de Barros et al. 2016). HOXD11 may affect

the progression of prostate cancer by encoding transcription factors (Hayano et al. 2016).

Moreover, HOXD11 may be involved in cell proliferation and angiogenesis-promoting pathways

as a cancer candidate gene in hemangioblastoma (Mehrian-Shai et al. 2016). Some studies have

also found that $H O X D 11$ can be used as a candidate cancer gene for the diagnosis of breast

cancer due to its high methylation levels (Miyamoto et al. 2005). Similarly, Cai et al. showed

that $H O X D 11$ can be used as a candidate biomarker in patients with ovarian cancer due to its

abnormal methylation (Cai et al. 2007). At the same time, studies have explored the role of

HOXD11 in regulating cellular levels in cancer cells. A study by Daniel et al. found that

HOXD11 was significantly expressed in Head and Neck Squamous Cell Carcinoma (HNSCC),

and knockdown of HOXD11 was able to reduce invasion in HNSCC (Sharpe et al. 2014).

HOXD11 may enhance cell growth, clonality, and metastatic potential in Ewing sarcoma (von

Heyking et al. 2016). Although a number of studies have suggested that $H O X D 11$ plays a crucial

role in cancer progression, the role of $H O X D 11$ in gliomas has not yet been elucidated.

We found that $H O X D 11$, as a novel carcinogenic gene in gliomas, expression levels were

significantly higher in gliomas than that in non-tumor brain tissues. This result is completely

credible and in line with the rigor of scientific research. Firstly, there are more than a thousand of

glioma tissue samples in this result, and the data types contain data from multiple races groups,

such as White, Asian and Black or African American. Secondly, the data used for analysis comes

Peer] reviewing PDF | (2020:09:53246:1:1:CHECK 15 Dec 2020) 
377 from a variety of analysis technologies including RNA sequencing, gene chip technology, RT-

378 qPCR and other technologies, which can complement each other and ensure the authenticity and

379 accuracy of the data. Third, the Kaplan-Meier method and Cox-regression analysis showed that

380 HOXD11 could lead to poor prognosis as an independent risk factor. Finally, it is worth

381

382

emphasizing that the knockdown of $H O X D 11$ can inhibit the proliferation and migration of U251 glioma cells. Therefore, we have sufficient reason to confirm that $H O X D 11$ can be a valuable biomarker for targeted treatment of glioma patients.

In order to reveal the mechanism of $H O X D 11$ leading to poor prognosis in glioma patients, GSEA was used to indirectly reveal the signal pathway activated by $H O X D 11$, which indicated that $H O X D 11$ is significantly enriched in cancer-related cell signaling pathways, such as cell cycle, DNA replication, ECM receptor interaction, and focal adhesion. As one of the most important cell signaling pathways, the cell cycle plays an extremely crucial role in the development of various diseases, especially the progression of cancer. Studies have shown that mutations in genes that regulate the cell cycle may be associated with glioma progression, recurrence, and poor prognosis in patients with gliomas (Jonsson et al. 2019). Moreover, Zhou et al. showed that knockdown of Tripartite Motif containing 44 (TRIM44) can affect glioma cell growth by influencing cell cycle regulation (Zhou et al. 2019). Studies have shown that Bromodomain containing 4 (BRD4) may affect the proliferation and apoptosis of glioma cells through affecting DNA replication in the glioma cell line U251 (Du et al. 2018). Focal adhesion plays a crucial role in the development of tumors by affecting cell adhesion and migration (Paluch et al. 2016). It has been reported that Methyl Gallate (MG) is able to inhibit the 

417 glioma.

\section{Conclusions}

migration ability and activity of glioma cells by inhibiting the formation of focal adhesion (Lee et al. 2013). In glioma cells, changes in Glial Fibrillary Acidic Protein (GFAP) can affect the invasiveness of glioma cells by affecting the expression of the Dual-Specificity Phosphatase 4 (DUSP4) in focal adhesions (van Bodegraven et al. 2019). In combination with the above studies, we believe that $H O X D 11$ may play a role in the aforementioned signaling pathway and thus affect the activity, adhesion, proliferation, and migration of glioma cells.

Although we have incorporated both the data sources of diverse for analysis, which contains Chinese, African American, and Caucasian individuals, so that the differences observed may not be narrowly limited to one ethnicity. Nonetheless, this research still has certain shortcomings to be improved. For example: based on the WHO glioma classification in 2016, more attention has been paid to the molecular typing of glioma. However, most of our data were obtained from public databases which may not necessarily contain information on the molecular typing of gliomas. Therefore, if conditions permit, we may develop more detailed research programs related to the molecular typing of gliomas. As another limitation, the mechanism whereby high expression of $H O X D 11$ leads to poor prognosis in glioma patients was indirectly revealed by functional enrichment analysis via GSEA. We only confirmed that HOXD11 knockdown could block U251 cells to G2 phase by flow cytometry and western blotting. Therefore, other specific mechanisms of $H O X D 11$ affecting glioma need to be further verified by other research. This study only provides a clue to lead more researchers to pay attention to the role of $H O X D 11$ in 
419 This study found that compared with normal controls, the expression level of HOXD11 was

420 higher in glioma samples at the cell and tissue level, and may be related to the poor overall

421 survival of patients with glioma. In addition, HOXD11 may be involved in cancer-related cell

422 signaling pathways, such as cell cycle, DNA replication, focal adhesion, and ECM receptor

423

424

425

426

427

428

429

430

431

432

433

434

435

436

437

438

439

440

441

442

443

444

445

446

447

interaction, and thereby affect the malignant progression of glioma by participating in the

regulation of the cell cycle. Therefore, $H O X D 11$ could be used as a candidate biomarker for

molecular therapy and prognostic evaluation of patients with glioma.

\section{Acknowledgements}

We appreciate the support of Affiliated of Henan Provincial People's Hospital.

\section{References}

Abbaoui B, Telu KH, Lucas CR, Thomas-Ahner JM, Schwartz SJ, Clinton SK, Freitas MA, and Mortazavi A. 2017. The impact of cruciferous vegetable isothiocyanates on histone acetylation and histone phosphorylation in bladder cancer. J Proteomics 156:94-103. 10.1016/j.jprot.2017.01.013

Cai LY, Abe M, Izumi S, Imura M, Yasugi T, and Ushijima T. 2007. Identification of PRTFDC1 silencing and aberrant promoter methylation of GPR150, ITGA8 and HOXD11 in ovarian cancers. Life Sci 80:1458-1465. 10.1016/j.lfs.2007.01.015

Dawson MA, and Kouzarides T. 2012. Cancer epigenetics: from mechanism to therapy. Cell 150:12-27. 10.1016/j.cell.2012.06.013

de Barros ELBR, Ramao A, Pinheiro DG, Alves CP, Kannen V, Jungbluth AA, de Araujo LF, Muys BR, Fonseca AS, Placa JR, Panepucci RA, Neder L, Saggioro FP, Mamede RC, Figueiredo DL, and Silva WA, Jr. 2016. HOX genes: potential candidates for the progression of laryngeal squamous cell carcinoma. Tumour Biol 37:1508715096. 10.1007/s13277-016-5356-8

Du Z, Song X, Yan F, Wang J, Zhao Y, and Liu S. 2018. Genome-wide transcriptional analysis of BRD4-regulated genes and pathways in human glioma U251 cells. Int J Oncol. 10.3892/ijo.2018.4324

Feng E, Liang T, Wang X, Du J, Tang K, Wang X, Wang F, and You G. 2019. Correlation of alteration of HLA-F expression and clinical characterization in 593 brain glioma samples. J Neuroinflammation 16:33. 10.1186/s12974-019-1418-3

Peer] reviewing PDF | (2020:09:53246:1:1:CHECK 15 Dec 2020) 
Grzmil M, Morin P, Jr., Lino MM, Merlo A, Frank S, Wang Y, Moncayo G, and Hemmings BA. 2011. MAP kinaseinteracting kinase 1 regulates SMAD2-dependent TGF- $\beta$ signaling pathway in human glioblastoma. Cancer Res 71:2392-2402. 10.1158/0008-5472.Can-10-3112

Guan X, Zhang C, Zhao J, Sun G, Song Q, and Jia W. 2018. CMTM6 overexpression is associated with molecular and clinical characteristics of malignancy and predicts poor prognosis in gliomas. EBioMedicine 35:233-243. 10.1016/j.ebiom.2018.08.012

Harada H, Miyamaoto K, Kimura M, Ishigami T, Taniyama K, and Okada M. 2019. Lung cancer risk stratification using methylation profile in the oral epithelium. Asian Cardiovasc Thorac Ann 27:87-92. $10.1177 / 0218492318813443$

Hayano T, Matsui H, Nakaoka H, Ohtake N, Hosomichi K, Suzuki K, and Inoue I. 2016. Germline Variants of Prostate Cancer in Japanese Families. PLoS One 11:e0164233. 10.1371/journal.pone.0164233

Jablonska E, and Reszka E. 2017. Selenium and Epigenetics in Cancer: Focus on DNA Methylation. Adv Cancer Res 136:193-234. 10.1016/bs.acr.2017.07.002

Jonsson P, Lin AL, Young RJ, DiStefano NM, Hyman DM, Li BT, Berger MF, Zehir A, Ladanyi M, Solit DB, Arnold AG, Stadler ZK, Mandelker D, Goldberg ME, Chmielecki J, Pourmaleki M, Ogilvie SQ, Chavan SS, McKeown AT, Manne M, Hyde A, Beal K, Yang TJ, Nolan CP, Pentsova E, Omuro A, Gavrilovic IT, Kaley TJ, Diamond EL, Stone JB, Grommes C, Boire A, Daras M, Piotrowski AF, Miller AM, Gutin PH, Chan TA, Tabar VS, Brennan CW, Rosenblum M, DeAngelis LM, Mellinghoff IK, and Taylor BS. 2019. Genomic Correlates of Disease Progression and Treatment Response in Prospectively Characterized Gliomas. Clin Cancer Res 25:5537-5547. 10.1158/1078-0432.Ccr-19-0032

Kim K, Lee K, Bang H, Kim JY, and Choi JK. 2016. Intersection of genetics and epigenetics in monozygotic twin genomes. Methods 102:50-56. 10.1016/j.ymeth.2015.10.020

Kondo Y, Shinjo K, and Katsushima K. 2017. Long non-coding RNAs as an epigenetic regulator in human cancers. Cancer Sci 108:1927-1933. 10.1111/cas.13342

Kong Z, Wang Y, and Ma W. 2018. Vaccination in the immunotherapy of glioblastoma. Hum Vaccin Immunother 14:255-268. 10.1080/21645515.2017.1388481

Lee SH, Kim JK, Kim DW, Hwang HS, Eum WS, Park J, Han KH, Oh JS, and Choi SY. 2013. Antitumor activity of methyl gallate by inhibition of focal adhesion formation and Akt phosphorylation in glioma cells. Biochim Biophys Acta 1830:4017-4029. 10.1016/j.bbagen.2013.03.030

Liang R, Zhi Y, Zheng G, Zhang B, Zhu H, and Wang M. 2019. Analysis of long non-coding RNAs in glioblastoma for prognosis prediction using weighted gene co-expression network analysis, Cox regression, and L1LASSO penalization. Onco Targets Ther 12:157-168. 10.2147/ott.S171957

Mehrian-Shai R, Yalon M, Moshe I, Barshack I, Nass D, Jacob J, Dor C, Reichardt JK, Constantini S, and Toren A. 2016. Identification of genomic aberrations in hemangioblastoma by droplet digital PCR and SNP microarray highlights novel candidate genes and pathways for pathogenesis. BMC Genomics 17:56. 10.1186/s12864016-2370-6

Miyamoto K, Fukutomi T, Akashi-Tanaka S, Hasegawa T, Asahara T, Sugimura T, and Ushijima T. 2005. Identification of 20 genes aberrantly methylated in human breast cancers. Int $J$ Cancer 116:407-414. $10.1002 / \mathrm{ijc} .21054$

Ostrom QT, Bauchet L, Davis FG, Deltour I, Fisher JL, Langer CE, Pekmezci M, Schwartzbaum JA, Turner MC, Walsh KM, Wrensch MR, and Barnholtz-Sloan JS. 2014. The epidemiology of glioma in adults: a "state of 
489

490

491

492

493

494

495

496

497

498

499

500

501

502

503

504

505

506

507

508

509

510

511

512

513

514

515

516

517

518

519

520

521

522

523

524

525

526

the science" review. Neuro Oncol 16:896-913. 10.1093/neuonc/nou087

Paluch EK, Aspalter IM, and Sixt M. 2016. Focal Adhesion-Independent Cell Migration. Annu Rev Cell Dev Biol 32:469-490. 10.1146/annurev-cellbio-111315-125341

Rodini CO, Xavier FC, Paiva KB, De Souza Setubal Destro MF, Moyses RA, Michaluarte P, Carvalho MB, Fukuyama EE, Tajara EH, Okamoto OK, and Nunes FD. 2012. Homeobox gene expression profile indicates HOXA5 as a candidate prognostic marker in oral squamous cell carcinoma. Int $J$ Oncol 40:1180-1188. 10.3892/ijo.2011.1321

Sharpe DJ, Orr KS, Moran M, White SJ, McQuaid S, Lappin TR, Thompson A, and James JA. 2014. POU2F1 activity regulates HOXD10 and HOXD11 promoting a proliferative and invasive phenotype in head and neck cancer. Oncotarget 5:8803-8815. 10.18632/oncotarget.2492

Sun L, Hui AM, Su Q, Vortmeyer A, Kotliarov Y, Pastorino S, Passaniti A, Menon J, Walling J, Bailey R, Rosenblum M, Mikkelsen T, and Fine HA. 2006. Neuronal and glioma-derived stem cell factor induces angiogenesis within the brain. Cancer Cell 9:287-300. 10.1016/j.ccr.2006.03.003

Taal W, Bromberg JE, and van den Bent MJ. 2015. Chemotherapy in glioma. CNS Oncol 4:179-192. 10.2217/cns.15.2

Tang Z, Li C, Kang B, Gao G, Li C, and Zhang Z. 2017. GEPIA: a web server for cancer and normal gene expression profiling and interactive analyses. Nucleic Acids Res 45:W98-W102. 10.1093/nar/gkx247

Tomczak K, Czerwinska P, and Wiznerowicz M. 2015. The Cancer Genome Atlas (TCGA): an immeasurable source of knowledge. Contemp Oncol (Pozn) 19:A68-77. 10.5114/wo.2014.47136

van Bodegraven EJ, van Asperen JV, Sluijs JA, van Deursen CBJ, van Strien ME, Stassen O, Robe PAJ, and Hol EM. 2019. GFAP alternative splicing regulates glioma cell-ECM interaction in a DUSP4-dependent manner. FASEB J 33:12941-12959. 10.1096/fj.201900916R

von Heyking K, Roth L, Ertl M, Schmidt O, Calzada-Wack J, Neff F, Lawlor ER, Burdach S, and Richter GH. 2016. The posterior HOXD locus: Its contribution to phenotype and malignancy of Ewing sarcoma. Oncotarget 7:41767-41780. 10.18632/oncotarget.9702

Wang W, Zhao Z, Wu F, Wang H, Wang J, Lan Q, and Zhao J. 2018. Bioinformatic analysis of gene expression and methylation regulation in glioblastoma. $J$ Neurooncol 136:495-503. 10.1007/s11060-017-2688-1

Xu W, Lu J, Zhao Q, Wu J, Sun J, Han B, Zhao X, and Kang Y. 2019. Genome-Wide Plasma Cell-Free DNA Methylation Profiling Identifies Potential Biomarkers for Lung Cancer. Dis Markers 2019:4108474. $10.1155 / 2019 / 4108474$

Zavadskaya capital Te C. 2015. Photodynamic therapy in the treatment of glioma. Exp Oncol 37:234-241.

Zhang E, Han L, Yin D, He X, Hong L, Si X, Qiu M, Xu T, De W, Xu L, Shu Y, and Chen J. 2017. H3K27 acetylation activated-long non-coding RNA CCAT1 affects cell proliferation and migration by regulating SPRY4 and HOXB13 expression in esophageal squamous cell carcinoma. Nucleic Acids Res 45:3086-3101. 10.1093/nar/gkw1247

Zhou X, Yang Y, Ma P, Wang N, Yang D, Tu Q, Sun B, Xiang T, Zhao X, Hou Z, and Fang X. 2019. TRIM44 is indispensable for glioma cell proliferation and cell cycle progression through AKT/p21/p27 signaling pathway. J Neurooncol. 10.1007/s11060-019-03301-0

Peer] reviewing PDF | (2020:09:53246:1:1:CHECK 15 Dec 2020) 


\section{Table $\mathbf{1}$ (on next page)}

The gene set enriches the high HOXD11 expression phenotype. 
1

2 Table 1. The gene set enriches the high HOXD11 expression phenotype.

\begin{tabular}{lllc}
\hline Gene set name & NES & NOM p-val & FDR q-val \\
\hline KEGG-CELL CYCLE & 1.728216 & 0.023 & 0.117 \\
KEGG-DNA REPLICATION & 1.6110542 & 0.048 & 0.194 \\
KEGG-ECM RECEPTOR INTERACTION & 1.8454952 & 0.011 & 0.177 \\
KEGG-FOCAL ADHESION & 1.7667084 & 0.013 & 0.118 \\
\hline
\end{tabular}

4 NOM P-value $<0.05$ and FDR q-value $<0.25$ were considered as significantly enriched.

5 


\section{Figure 1}

The higher expression level of HOXD11 in glioma tissues and cells.

(a) HOXD11 was highly expressed in glioblastoma (GBM) tissues $(n=163)$ relative to normal brain tissues( $n=207$ ) from GEPIA database; (b) The expression level of HOXD11 in glioma tissues ( $n=23$ ) and non-tumor brain tissues ( $n=77)$ in GSE4290; (c) The expression level of HOXD11 in glioma cell lines ( $n=2$ in LN319, $n=2$ in LN229, $n=2$ in BS149 and $n=2$ in LN018) and human-derived astrocyte ( $n=3$ in HA) in GSE15824; (d) RT-qPCR in glioma tissues ( $n=9)$ and non-tumor brain tissues $(n=5)$ collected from clinical; (e) RT-qPCR in glioma cell lines ( LN229,T98 and U251) and human-derived astrocyte (HA). (c-e) These graphs show the difference in the expression of HOXD11 at the mRNA level, and are represented by $*(p<0.05), * *(p<0.01), * * *(p<0.001)$ or $* * * *(p<0.001)$ Statistical differences determined by one-way ANOVA. 
a

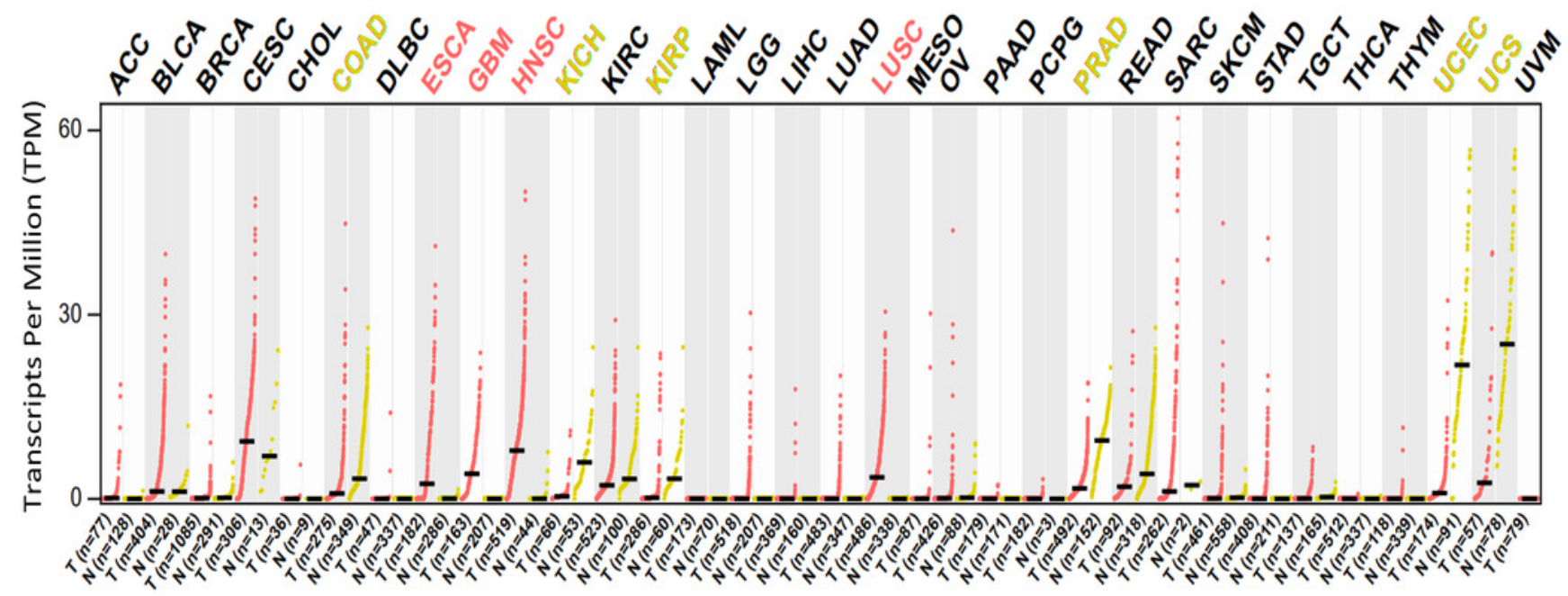

b

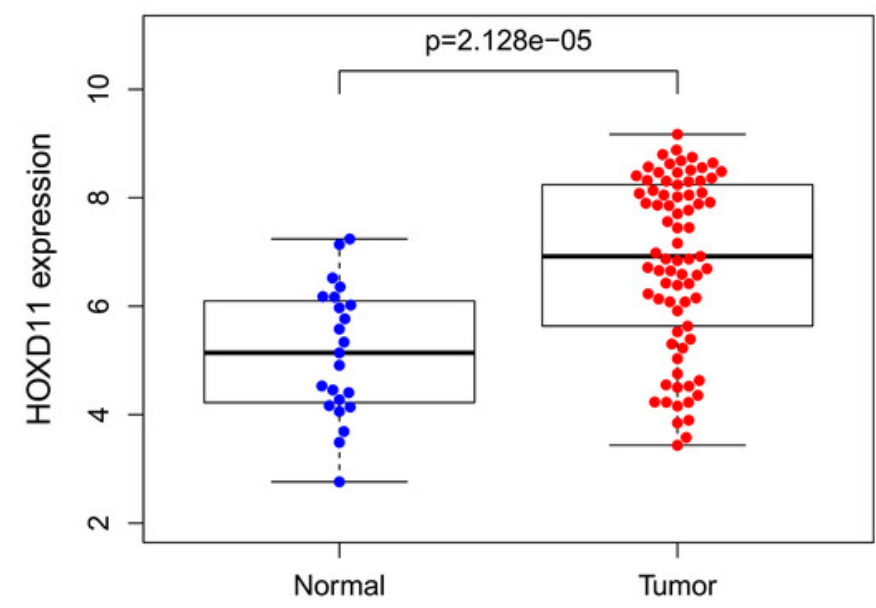

d

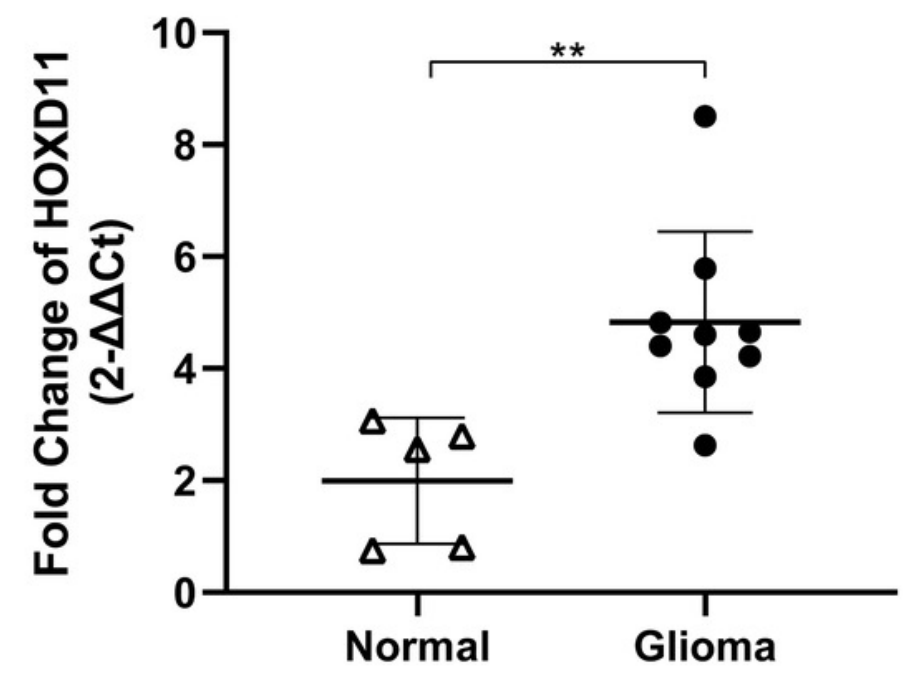

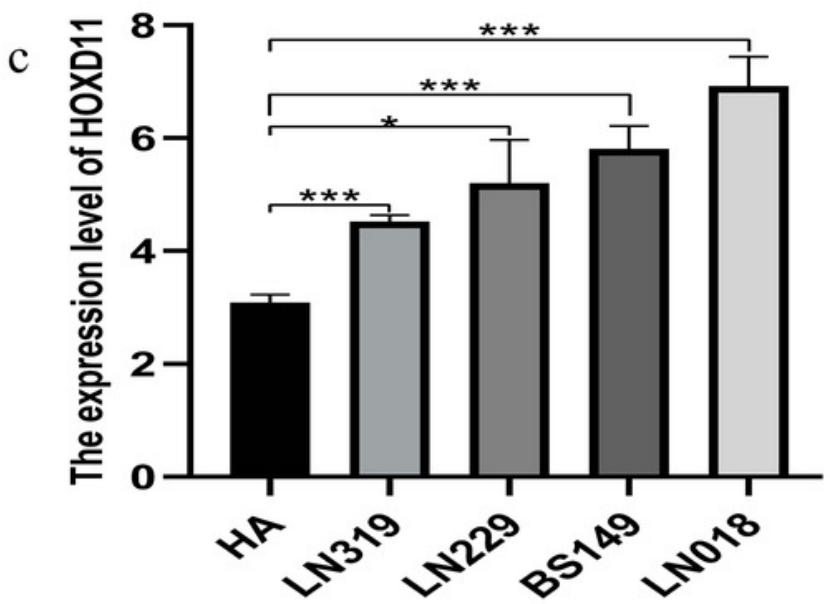

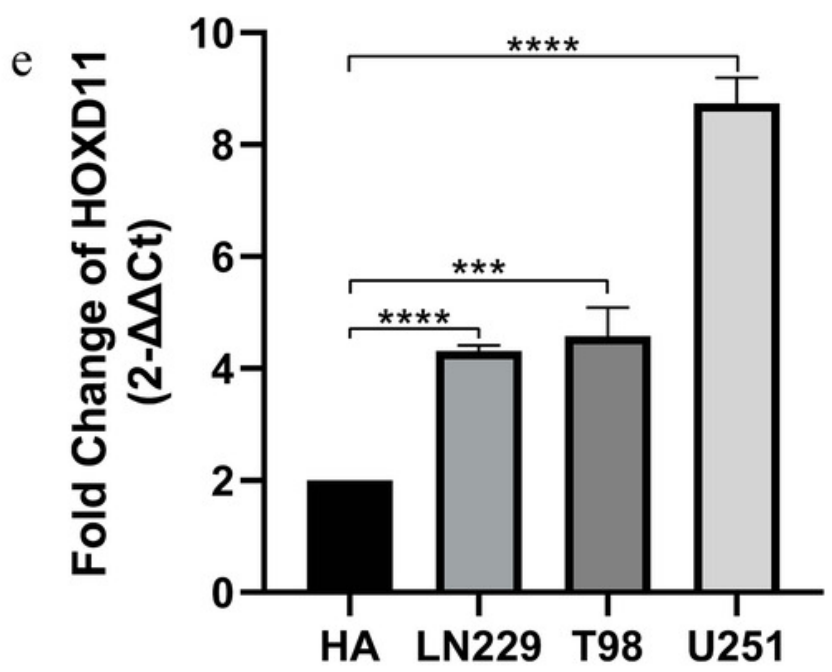




\section{Figure 2}

The relationship between the difference of HOXD11 expression level and various clinical characteristics.

The various clinical characteristics include (a):PRS type, (b): Grade, (c): Chemo status, (d): Age, (e): 1p19q codeletion status, (f): IDH mutation status, (g): Histology. A: strocytoma; AA: anaplastic astrocytoma; AO: anaplastic oligodendroglioma; AOA: anaplastic oligoastrocytoma; GBM: glioblastoma multiforme; O: oligodendroglioma; OA: oligoastrocytoma; rA: recurrence of strocytoma; rAA: recurrence of anaplastic astrocytoma; rAO: recurrence of anaplastic oligodendroglioma; rAOA: recurrence of anaplastic oligoastrocytoma; rGBM: recurrence of glioblastoma multiforme; rO: recurrence of oligodendroglioma; rOA: recurrence of oligoastrocytoma; sGBM: secondary glioblastoma. 

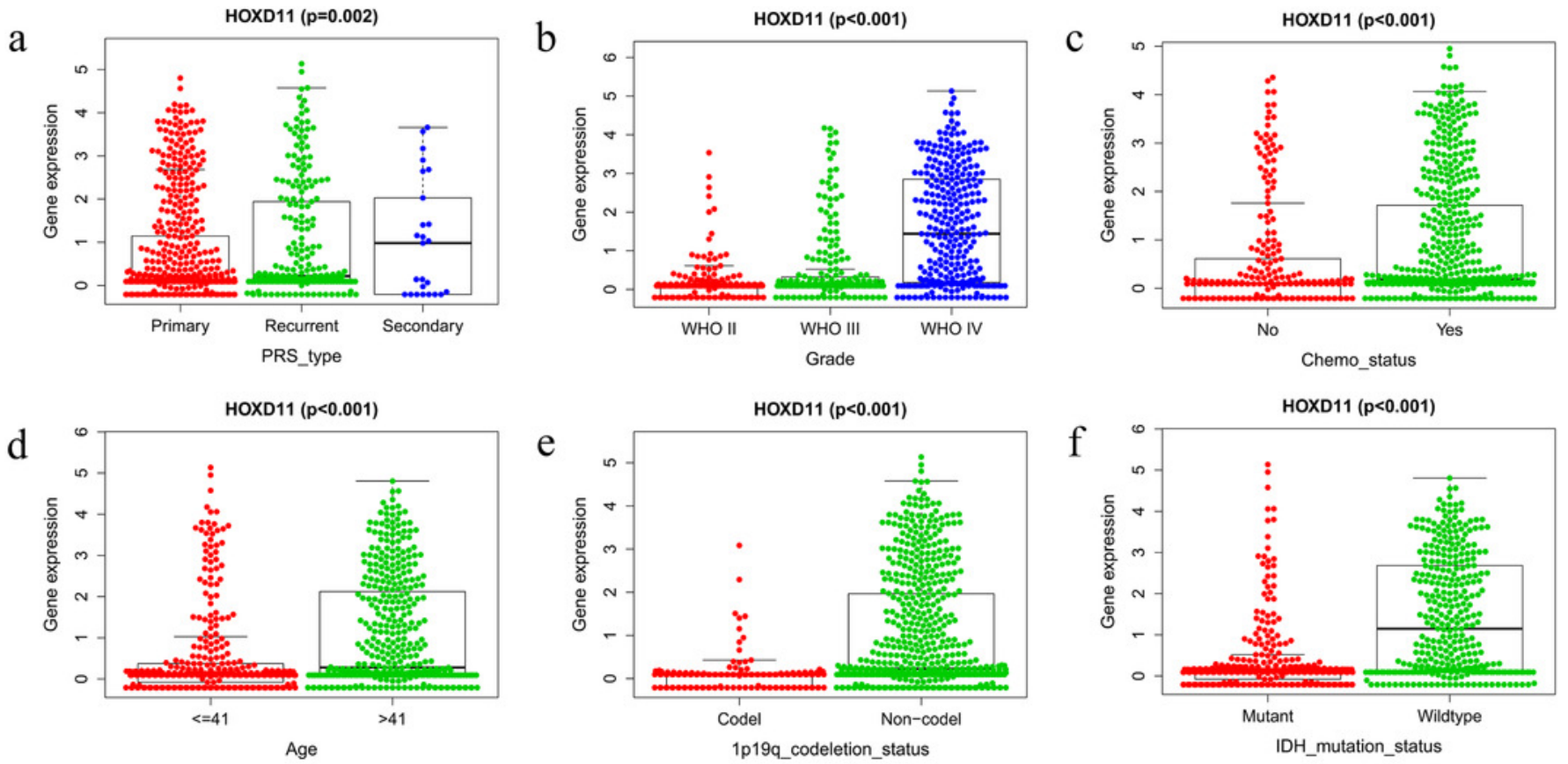

HOXD11 $(p<0.001)$

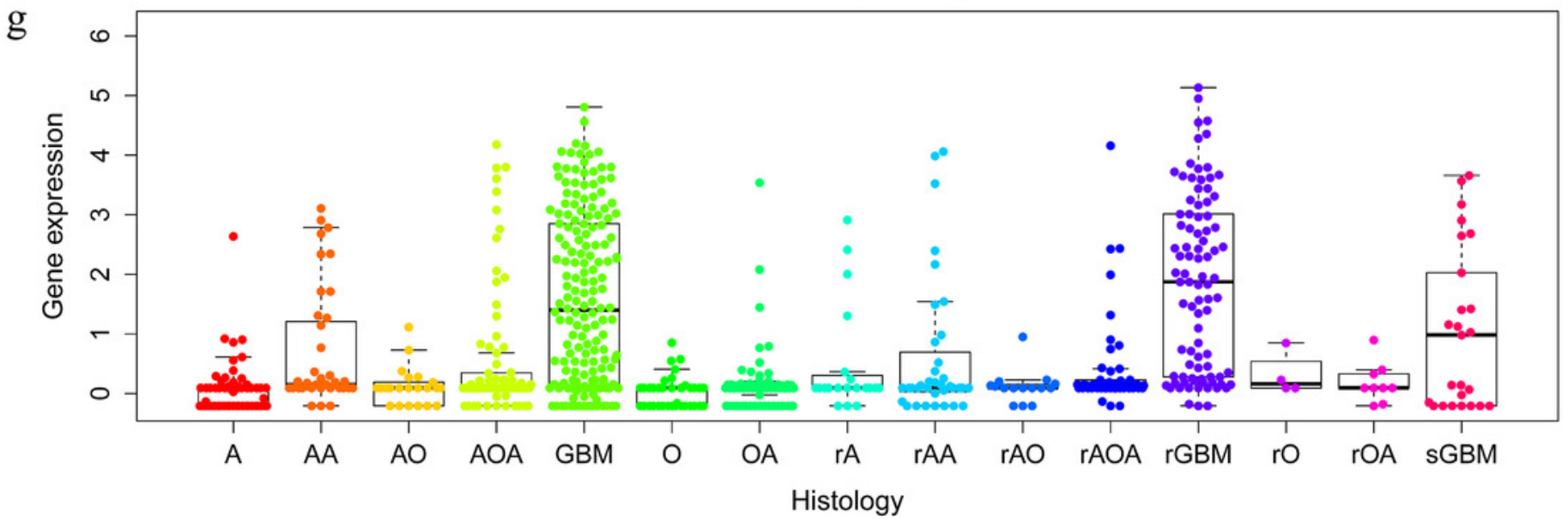


Figure 3

The relationship between the difference of the expression level of HOXD11 and Overall Survival (OS).

(a) CGGA sequence; (b) TCGA sequence; (c) IDH mutation with 1p19q codeletion from CGGA sequence; (d) IDH mutation without 1p19q codeletion from CGGA sequence; (e) IDH wild type from CGGA sequence.

a
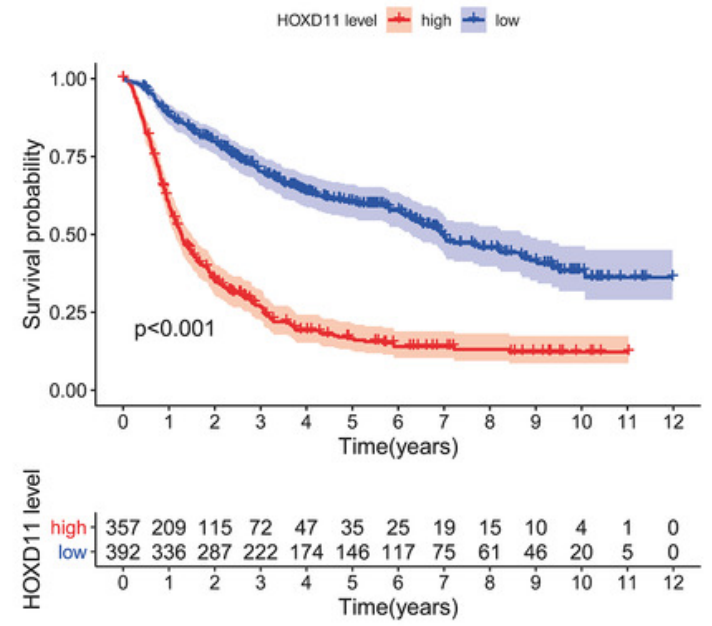

CGGA-sequence
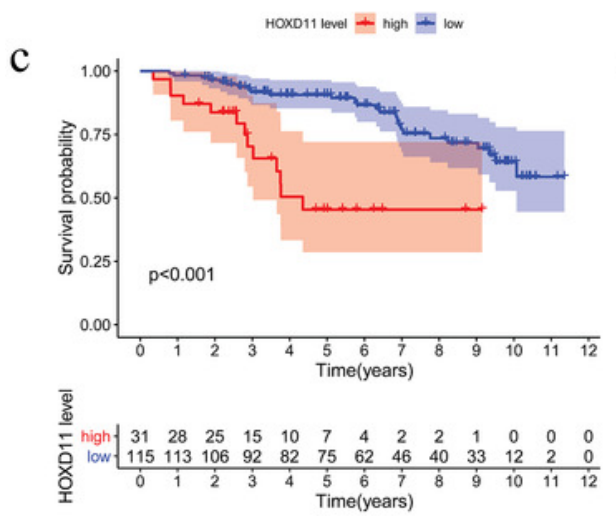

CGGA(IDH mutation with $1 \mathrm{p} 19 \mathrm{q}$ codeletion)

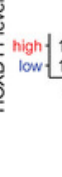

b
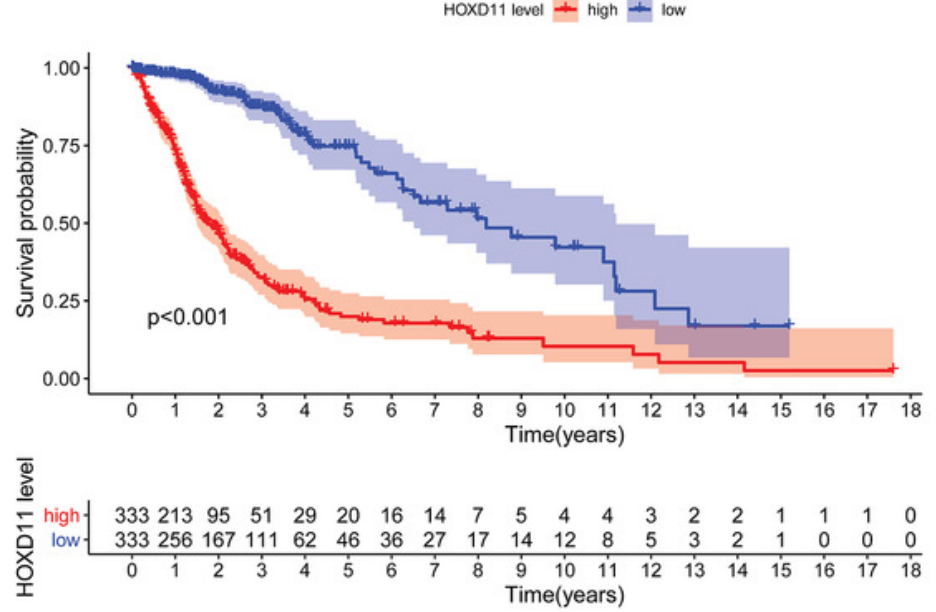

TCGA-sequence

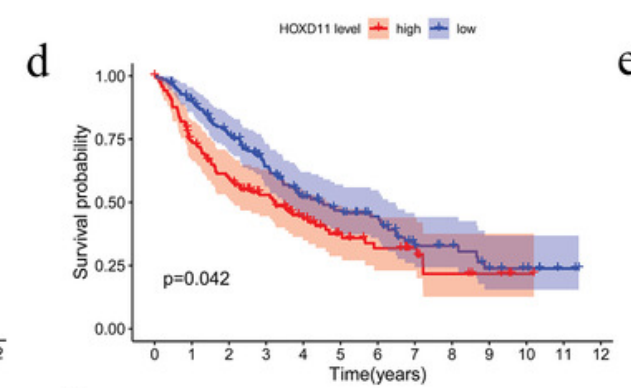

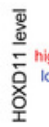

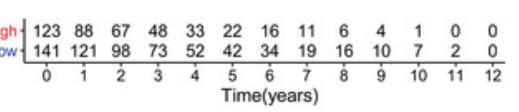

CGGA(IDH mutation without $1 \mathrm{p} 19 \mathrm{q}$ codeletion)
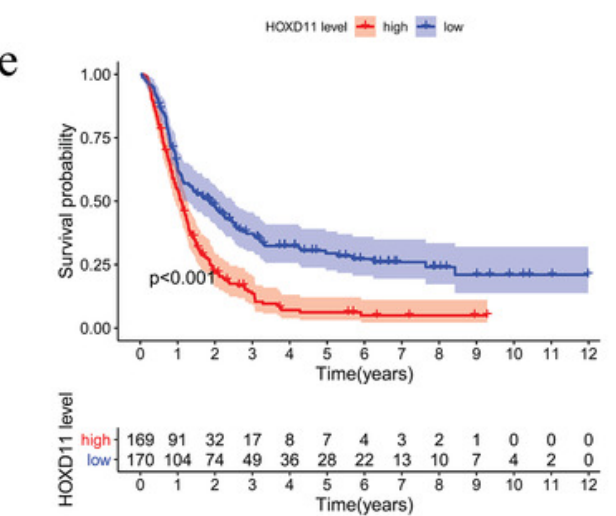

CGGA(IDH wild type) 
Figure 4

Prognostic factors and risk assessment of glioma and the diagnostic value of HOXD11.

(a) Univariate regression of prognostic in patients with glioma; (b) Multivariate survival model of prognostic in patients with glioma; (c) The receiver operating characteristic (ROC) curve in CGGA sequence; (d) The receiver operating characteristic (ROC) curve in TCGA sequence.

a

\begin{tabular}{lrr} 
& pvalue & $\begin{array}{r}\text { Hazard ratio } \\
\text { HOXD11 }\end{array}$ \\
\hline$<0.001$ & $1.550(1.456-1.650)$ \\
PRS_type & $<0.001$ & $2.123(1.818-2.478)$ \\
Histology & $<0.001$ & $4.487(3.695-5.449)$ \\
Grade & $<0.001$ & $2.883(2.526-3.291)$ \\
Gender & 0.655 & $1.044(0.866-1.258)$ \\
Age & $<0.001$ & $1.624(1.345-1.960)$ \\
Radio & 0.571 & $0.929(0.720-1.199)$ \\
Chemo & $<0.001$ & $1.647(1.328-2.044)$ \\
IDH_mutation & $<0.001$ & $0.317(0.262-0.384)$ \\
1p19q_codeletion & $<0.001$ & $0.231(0.169-0.315)$
\end{tabular}

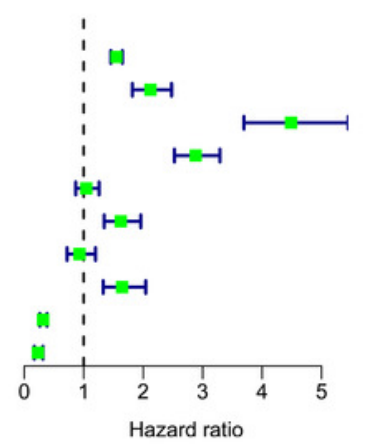

b
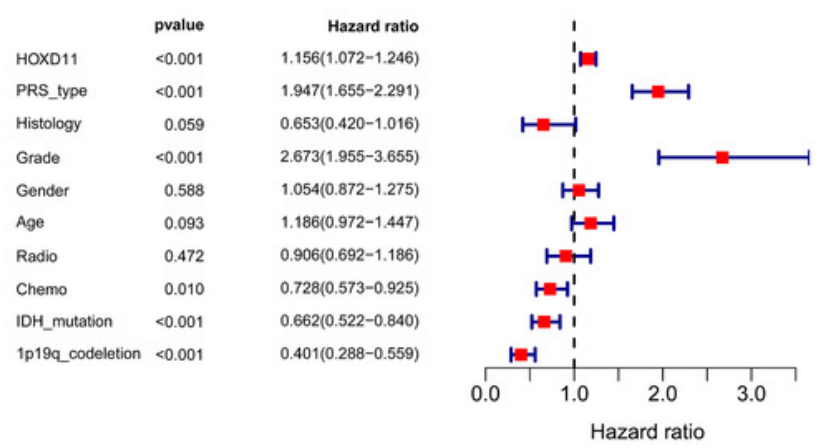

c

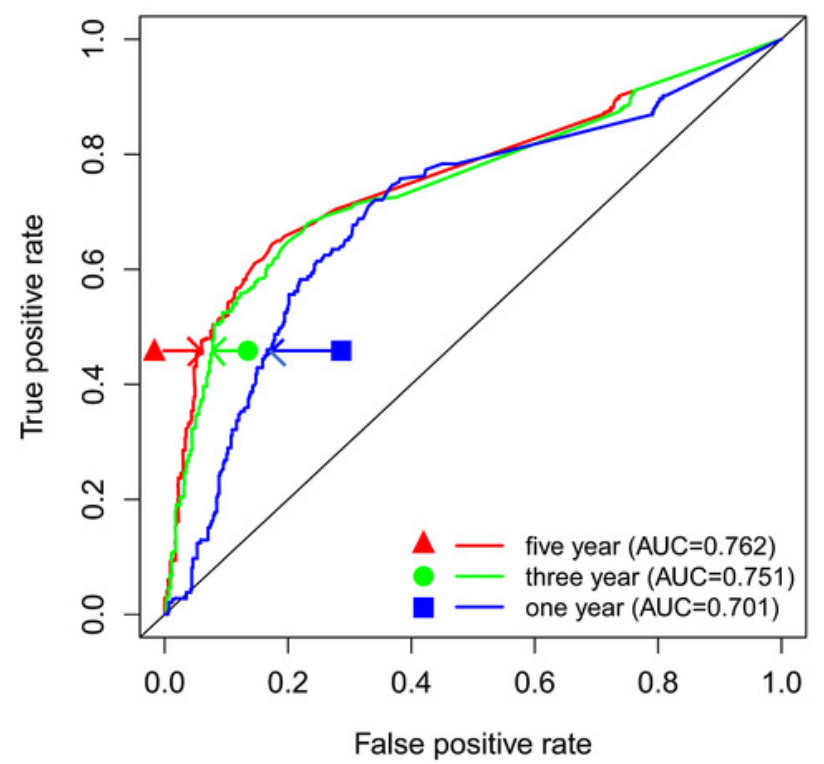

d

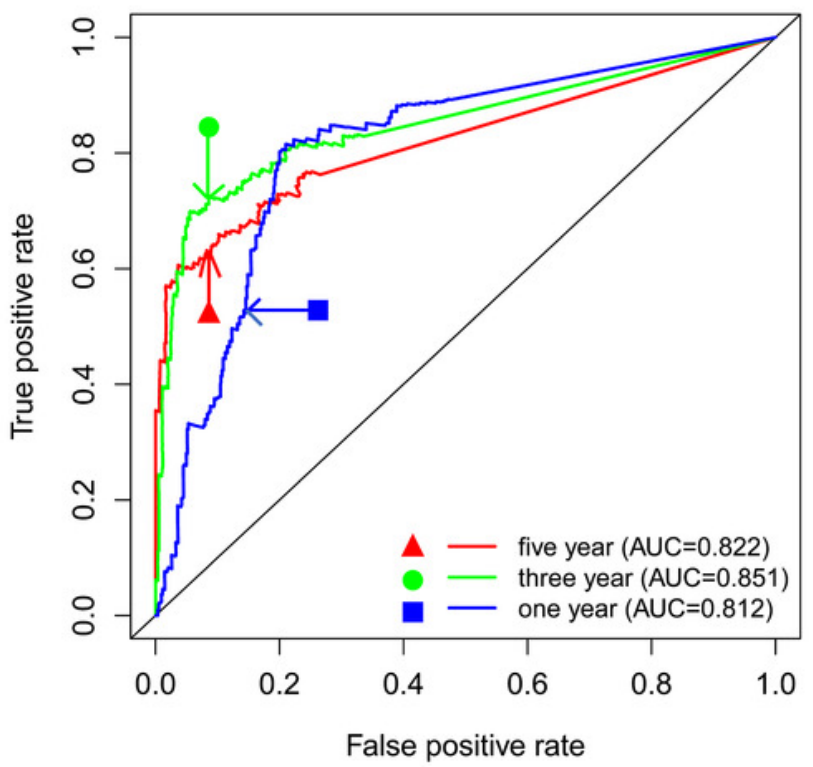




\section{Figure 5}

HOXD11 may be involved in promoting the proliferation and invasion of glioma.

(a) RT-qPCR after cell transfection; (b) colony formation assay after cell transfection; (c) KI67 Immunofluorescence staining after cell transfection; (d) statistical graph of KI67 immunofluorescence, $*(p<0.05)$; (e) MTT assay at $0 \mathrm{~h}, 12 \mathrm{~h}, 24 \mathrm{~h}, 48 \mathrm{~h}$ and $96 \mathrm{~h}$ after cell transfection, ${ }^{* *}(\mathrm{p}<0.01),{ }^{* * *}(\mathrm{p} \square 0.001) ;(\mathrm{f})$ Wound-healing assay at $0 \mathrm{~h}, 24 \mathrm{~h}, 48 \mathrm{~h}$ after cell transfection, ${ }^{* *}(p<0.01), * * *(p<0.001)$. 

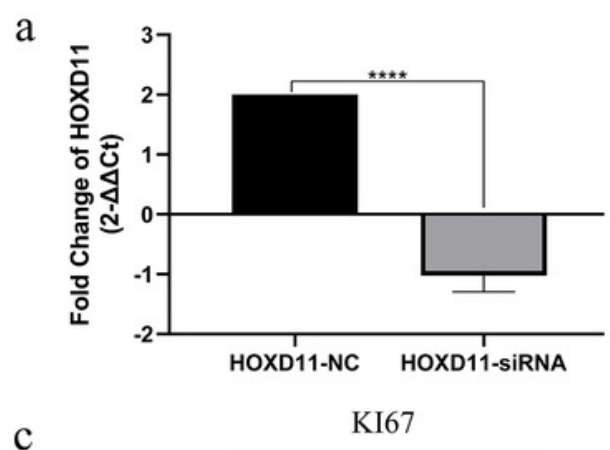

$\mathrm{c}$

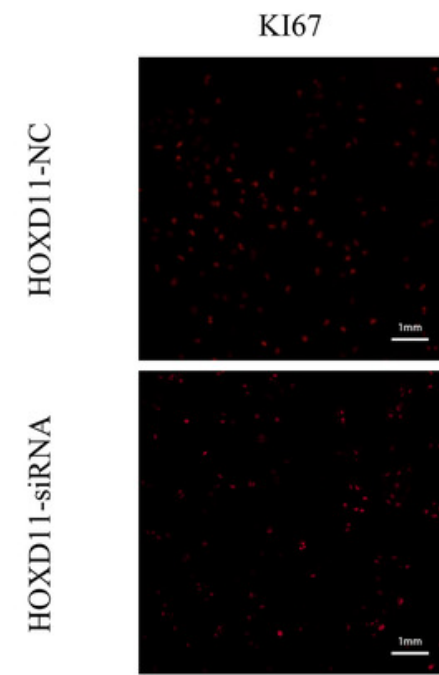

d

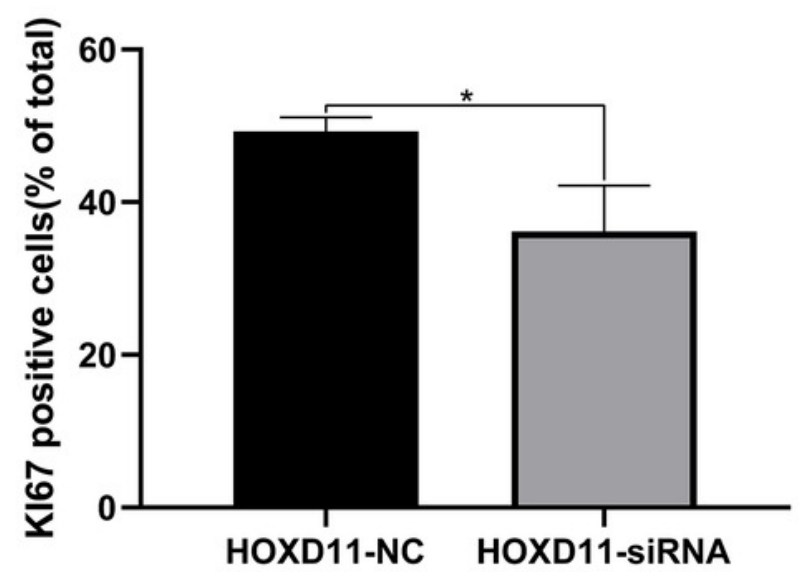

f

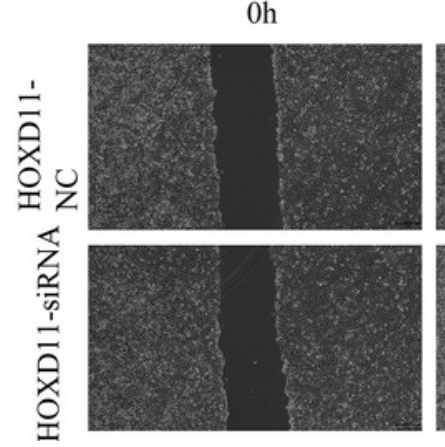

b

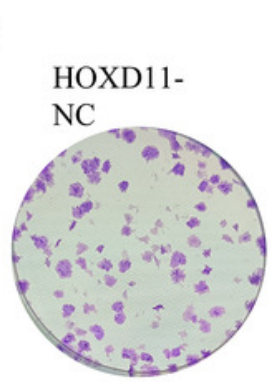

DAPI
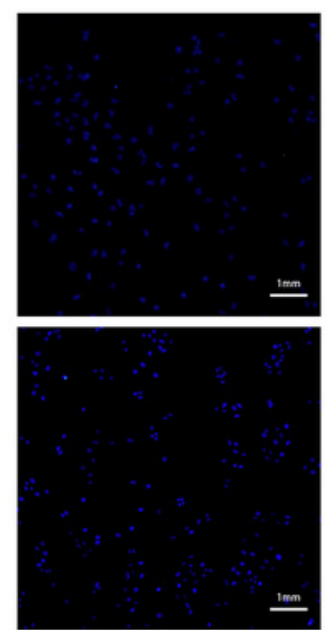

$24 \mathrm{~h}$

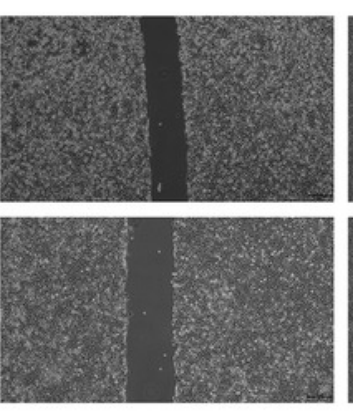

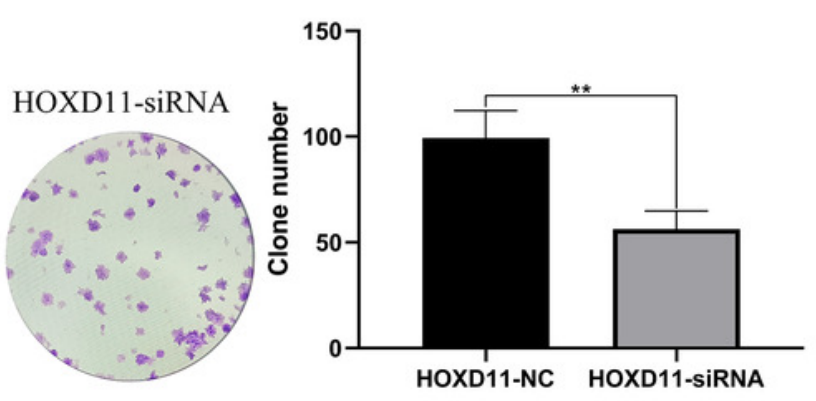

Merge
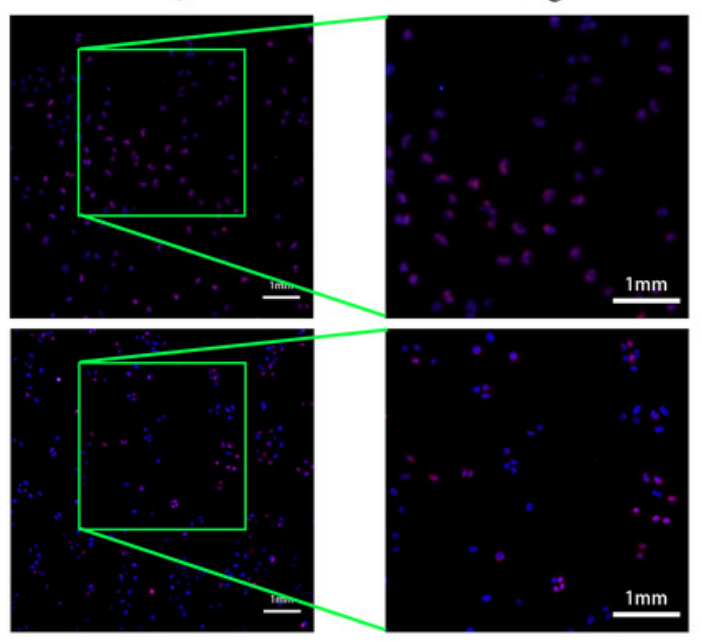

e

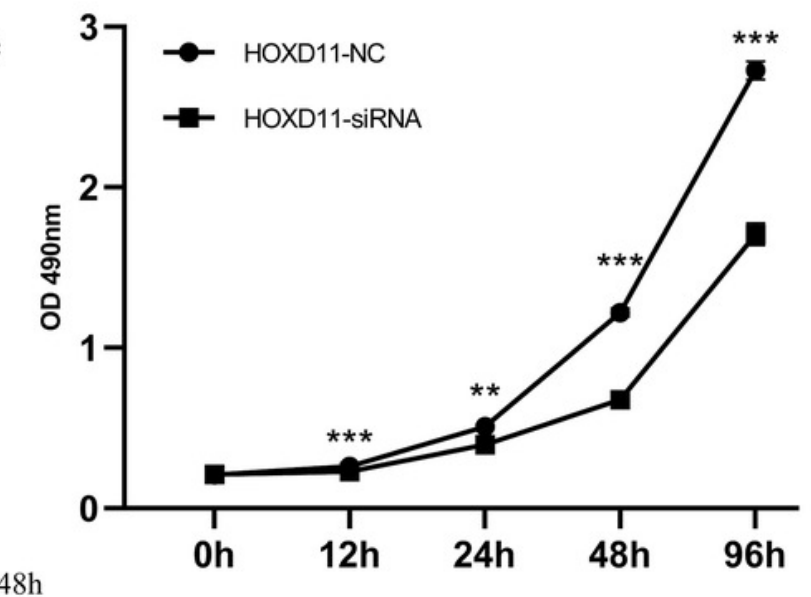
$48 \mathrm{~h}$
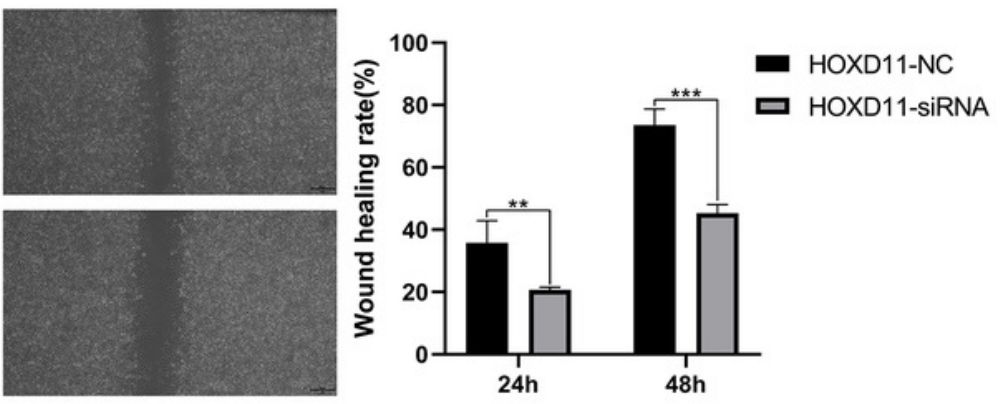


\section{Figure 6}

HOXD11 may be involved in regulating the $\mathrm{G} 2$ phase of the cell cycle in gliomas.

(a) Enrichment plots from GSEA. GSEA results showing cell cycle, DNA replication , ECM receptor interactions, focal adhesions differentially enriched in the high expression phenotype of HOXD11; (b) Cell cycle-related flow cytometry, ${ }^{* * *}$ ( $p<0.001$ ); (c) The expression level of cell cycle-related protein, GAPDH was used to confirm equal protein loading, $* * *(p<0.001)$. 
a
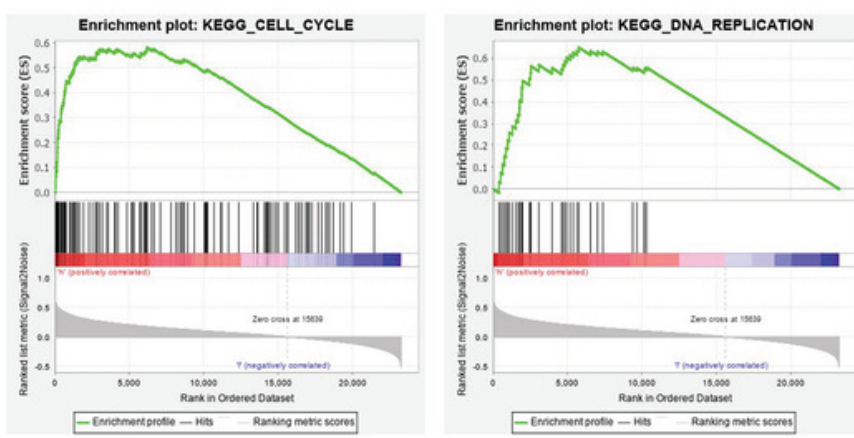

b

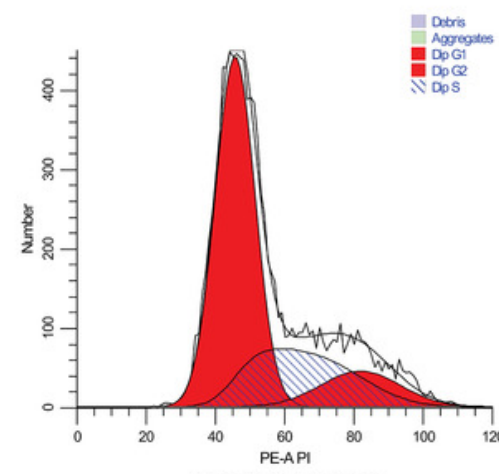

HOXD11-NC

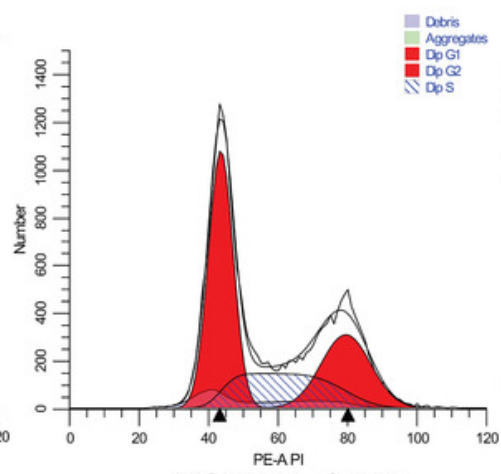

HOXD11-siRNA
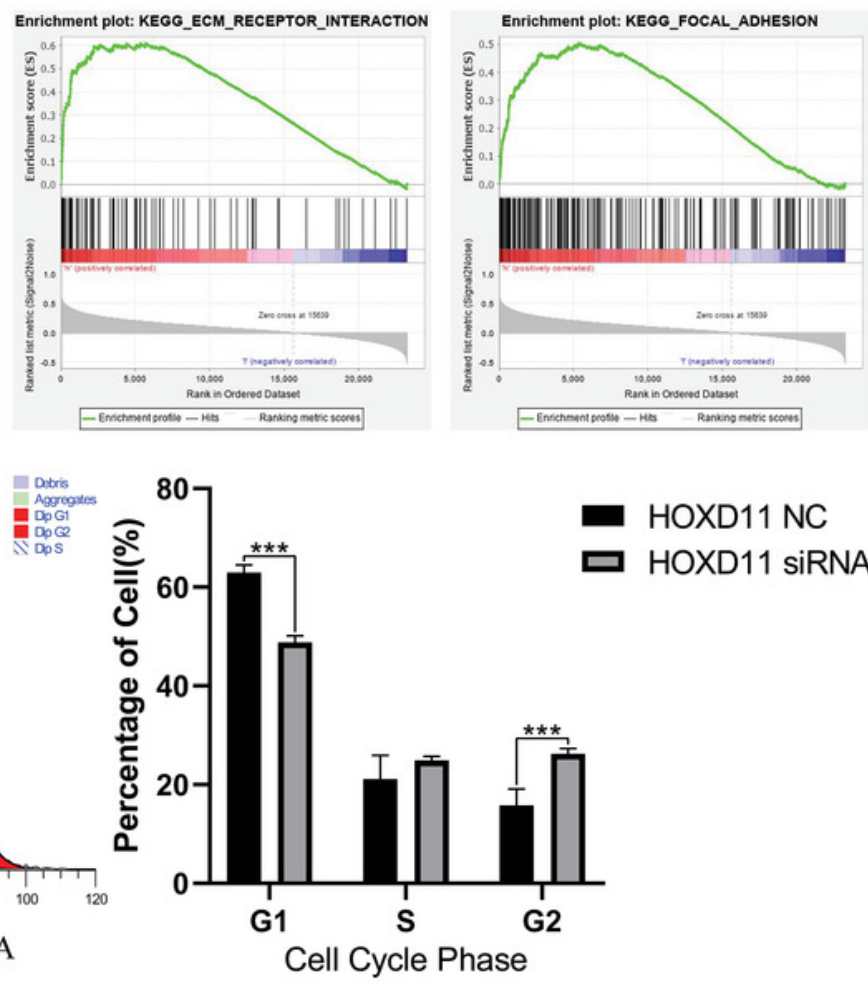

Cell Cycle Phase $\mathrm{c}$

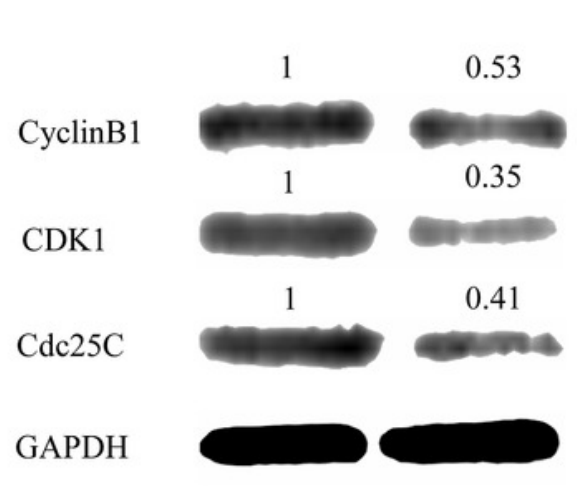



